\title{
Progenitor and explosion models of type la supernovae
}

\author{
Ataru Tanikawa* \\ Department of Earth Science and Astronomy, College of Arts and Sciences, The University of \\ Tokyo, 3-8-1 Komaba, Meguro-ku, Tokyo 153-8902, Japan \\ E-mail: tanikawa@ea.c.u-tokyo.ac.jp
}

\begin{abstract}
Type Ia supernovae (SNe Ia) are important objects in astronomy and astrophysics. They are one of the brightest and common transients in the universe, a standard candle for a cosmic distance, and a dominant origin of iron group elements. It is widely accepted that $\mathrm{SNe}$ Ia are thermodynamical explosions of carbon-oxygen (CO) white dwarfs (WDs) in binary systems. Nevertheless, it is yet unclear what the companion stars are, and what the masses of the exploding CO WDs are. Recently, many sub-Chandrasekhar mass explosions in the double-degenerate scenario have been suggested. We have assessed the violent merger and Dynamically-Driven Double-Degenerate Double-Detonation $\left(\mathrm{D}^{6}\right)$ models by numerical simulations. We have concluded that the violent merger model may not explain normal SNe Ia, since their circum-binary matter is much larger than the size of SN 2011fe, and their event rate is much smaller than the SN Ia rate in our Galaxy by an order of magnitude. However, it can be progenitors of peculiar SNe Ia. The $\mathrm{D}^{6}$ model has roughly consistent features with normal SNe Ia. However, the model has supernova ejecta with an asymmetric shape and low-velocity carbon-oxygen component due to the presence of the surviving companion $\mathrm{CO}$. If these features can be observed, the $\mathrm{D}^{6}$ model may be inconsistent with normal SNe Ia. In this case, the $\mathrm{D}^{6}$ model can be also progenitors of peculiar SNe Ia.
\end{abstract}

The Golden Age of Cataclysmic Variables and Related Objects V(GOLDEN2019)

2-7 September 2019

Palermo, Italy

${ }^{*}$ Speaker. 


\section{Introduction}

Type Ia supernovae (SNe Ia) play important roles in astronomy and astrophysics. They are one of the brightest and most common transients in the universe, are utilized as a cosmic standard candle, and can be a dominant source of iron group elements. There is wide consensus that they are powered by thermonuclear explosions of carbon oxygen (CO) white dwarfs (WDs) in binary systems [四. However, their progenitors have been controversial with respect to two points. The first point is the nature of the companion stars. The companion stars can be non-degenerate stars,

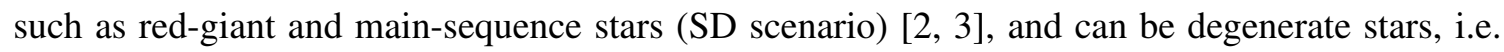
another WDs, (DD scenario) [ [⿴囗十, ] ] The second point is the mass of exploding WDs. It can be nearChandrasekhar mass [[]], and sub-Chandrasekhar mass [圆]. The progenitor problem of SNe Ia gets more complex due to the presence of not only SNe Ia used as a cosmic standard candle (hereafter, normal Ia), but also SNe Ia deviating from normal Ia (hereafter, peculiar Ia) [ [, , []].

Recent observations have greatly narrowed the allowed range of the SD scenario, although they cannot completely rule out the SD scenario. They have not found no red-giant stars in the pre-explosion images of SN 2011fe and SN 2014J [Q, ए0]. There is no main-sequence star in a supernova remnant LMC SNR [U]]. Theoretical studies have pointed out the difficulty of nearChandrasekhar mass explosion in the DD scenario. A merger remnant of two CO WDs finally results in an oxygen neon magnesium WD with super-Chandrasekhar mass [12], and collapses to a neutron star or black hole [[13]. In response to this situation, many sub-Chandrasekhar mass explosions in the DD scenario have been suggested: the violent merger model [14]], the collision model [ [15, [6, [7]], the Dynamically-Driven Double-Degenerate Double-Detonation $\left(\mathrm{D}^{6}\right)$ model

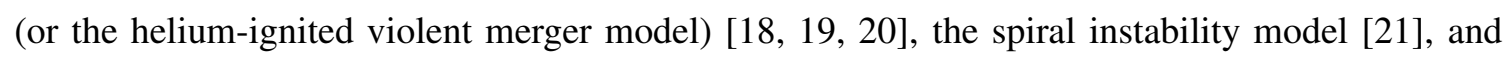
detached DD model [22]. In this paper, we discuss the violent merger and the $\mathrm{D}^{6}$ models.

The structure of this paper is as follows. In sections $\square$ and 1 , we investigate the violent merger and $\mathrm{D}^{6}$ models, respectively. In section 4 , we summarize this paper.

\section{The violent merger model}

We briefly explain the violent merger model. Let's consider a close binary system consisting of two CO WDs. They lose an orbital energy due to gravitational wave radiation. At some time, the lighter CO WD is tidally disrupted by gravity of the heavier CO WD. The debris of the lighter CO WD rapidly falls into the heavier CO WD. The rapid accretion forms a hot spot near the surface of the heavier CO WD. From the hot spot, $\mathrm{CO}$ detonation is generated, and incinerates the two CO WDs. Finally, the system is observed as an SN Ia.

We have assessed this model from two points of view by numerical simulation. The first point is whether this model can really succeed, and whether this model is observed as a normal Ia if it succeeds [[23]. The second point is whether the event rate of this model is sufficient for the normal Ia rate [24, 25]].

In order to assess the first point, we have thoroughly investigated a binary with 1.1 and $1.0 M_{\odot}$ CO WDs. We expect this binary succeeds in the violent merger explosion most easily. This is because these CO WDs have nearly the maximum mass of CO WDs, and mass accretion becomes more drastic with the mass ratio approaching to unity. We have performed four SPH simulations 
with 1.4, 2.8,5.5, and 11 million particles. All the simulations have more than 1 million particles, since the violent merger explosion does not become successful if the number of particles is less than a million [[26]. In each simulation, we have found a hot and dense spot enough to generate $\mathrm{CO}$ detonation. Moreover, the spot becomes hotter and more dense with the simulation increasing the number of particles. Thus, we have concluded that the violent merger explosion becomes successful in this binary system. We have examined the structure of this binary system at the moment when a hot spot to generate $\mathrm{CO}$ detonation appears. We have found circum-binary matter (CSM) is formed due to tidal disruption of the lighter CO WD. The CSM spreads to $\gtrsim 0.1 R_{\odot}$. Its size is too large to explain a normal Ia, such as SN $2011 \mathrm{fe}$ in which CSM size is inferred as $\lesssim 0.1 R_{\odot}$ [27, [28].

We have also performed SPH simulations for binary systems of two CO WDs with various mass combinations. The CO WD masses range from $0.5 M_{\odot}$ to $1.1 M_{\odot}$. For each mass combination, we have adopted different numbers of particles for convergence check. We have confirmed what mass combinations cannot ignite the violent merger explosion. We have found the violent merger becomes successful only when the lighter CO WDs have more than or equal to $0.8 M_{\odot}$. This is because mass accretion is not drastic enough to form a hot spot to generate $\mathrm{CO}$ detonation. This means that the event rate of the violent merger explosion is less than $10 \%$ of the normal Ia rate in our Galaxy.

In summary, we have concluded that the violent merger model cannot explain normal Ia for two reasons. The first reason is that the CSM of the violent merger model is too large for a normal Ia. The second reason is that the event rate of the violent merger model is less than the normal Ia rate by an order of magnitude. Nevertheless, the violent merger model may be progenitors of peculiar Ia.

\section{The $\mathbf{D}^{6}$ model}

In the $\mathrm{D}^{6}$ model, the pathway to SN Ia explosion is as follows. There is a binary system consisting of a CO WD and a WD lighter than the CO WD. The WD can be helium (He) WD or CO WD with a He envelope. The binary system experiences mass transfer from the lighter WD to the heavier WD. The transferred matter consists of He. The mass transfer process finally ignite He detonation on the surface of the heavier WD, and subsequently trigger $\mathrm{CO}$ detonation near the center of the heavier WD through the double detonation mechanism [ [6, 29]. The CO detonation explodes the heavier WD, and the explosion is observed as an SN Ia. The lighter WD survives, is suddenly free from the gravity of the heavier WD, and results in a hypervelocity WD. Since such hypervelocity WDs have been discovered [ए[D], the $\mathrm{D}^{6}$ model becomes one of promising progenitor models.

We have assessed whether the $\mathrm{D}^{6}$ model can be observed as normal Ia. In particular, we have focused on interaction of the heavier WD explosion with the lighter WD. First, we have performed SPH simulation to follow the explosion of the heavier CO WD with $1.0 M_{\odot}$, and response of the lighter CO WD with $\left.0.6 M_{\odot}[B]\right]$. The SPH simulations are couple with nuclear reaction networks, and have several ten million particles owing to our massively parallel simulation code. Then, the supernova ejecta has an asymmetric shape, since the lighter WD acts as an obstacle. If this asymmetric shape survives until its supernova remnant phase, the $\mathrm{D}^{6}$ model may not be consistent with Ia supernova remnants. However, the asymmetric shape may be erased through interaction 
with interstellar medium. We will confirm this issue in near future. Moreover, the supernova ejecta strips materials from the the lighter WD. The stripped materials compose a low-velocity CO component in the supernova ejecta. If these materials can be observed, the $\mathrm{D}^{6}$ model may disagree with normal Ia. Even if these features can be observed, the $\mathrm{D}^{6}$ model can be progenitors of peculiar Ia. We will investigate whether these materials can be observed by radiative transfer calculations in near future.

We have also investigated whether the explosions of the heavier WDs induce the explosions of the lighter WDs by numerical simulations with various mass combinations of WDs [B]] . In all the cases, we have used a CO WD with $1.0 M_{\odot}$ for the heavier WDs. We have adopted different WDs for the lighter WDs. We have found that the lighter WDs explode in two cases. In the first case, a He WD with $0.45 M_{\odot}$ explodes through He detonation starting at the surface ignited by the explosion of the heavier WD. The system yields $\sim 0.8 M_{\odot}{ }^{56} \mathrm{Ni}$. However, such explosion occurs only when the separation between the heavier and lighter WDs is unrealistically close. The lighter WD should be disrupted by the tidal field of the heavier WD before the system reaches to such a configuration. In the second case, a CO WD with $0.9 M_{\odot}$ in total and $0.054 M_{\odot} \mathrm{He}$ outer shell explodes through double detonation mechanism. Note that the double detonation mechanism starts from He detonation of the He shell triggered by the explosion of the heavier WD. The system synthesizes $\sim 1.0 M_{\odot}{ }^{56} \mathrm{Ni}$. Although this explosion is quite interesting, the He shell is unrealistically thick. If the He shell mass is $0.009 M_{\odot}$, the double detonation mechanism in the lighter WD does not work. Eventually, the explosion of the heavier WD cannot induce the explosion of the lighter WD. These facts indirectly support the $\mathrm{D}^{6}$ model as normal Ia progenitors, since explosions of two WDs produce too much ${ }^{56} \mathrm{Ni}$ for normal Ia.

In summary, we have investigated the $\mathrm{D}^{6}$ model, focusing on the interaction of the heavier WD explosions with the lighter WDs. Due to the presence of the lighter WDs, the supernova ejecta have asymmetric shapes, and low-velocity CO components. These features may not be consistent with normal Ia. We will investigate these points in near future. On the other hand, the lighter WDs cannot experience explosions triggered by the explosions of the heavier WDs in normal configurations. This indirectly supports the $\mathrm{D}^{6}$ model as normal Ia progenitors.

\section{Summary}

SNe Ia are important transients, and however their progenitors have been unclear. Recently, many sub-Chandrasekhar mass explosion models in the DD scenario have been suggested. Among them, we have assessed the violent merger and $\mathrm{D}^{6}$ models. We have concluded that the violent merger cannot explain normal Ia, since it has too large CSM, and too small event rate. Nevertheless, it can be progenitors of peculiar Ia. The $\mathrm{D}^{6}$ model has supernova ejecta with an asymmetric shape and low-velocity $\mathrm{CO}$ component. If these features can be observed, the $\mathrm{D}^{6}$ model may not be consistent with normal Ia. In this case, it can be progenitors of peculiar Ia. We will confirm them in near future.

\section{References}

[1] F. Hoyle and W. A. Fowler, Nucleosynthesis in Supernovae, The Astrophysical Journal 132 (1960) 565. 
[2] J. Whelan and I. Iben, Jr., Binaries and Supernovae of Type I, The Astrophysical Journal 186 (1973) 미․

[3] K. Nomoto, F.-K. Thielemann and K. Yokoi, Accreting white dwarf models of Type I supernovae. III Carbon deflagration supernovae, The Astrophysical Journal 286 (1984) 644.

[4] I. Iben, Jr. and A. V. Tutukov, Supernovae of type I as end products of the evolution of binaries with components of moderate initial mass (M not greater than about 9 solar masses), The Astrophysical Tournal Supplements 54(1984) 335.

[5] R. F. Webbink, Double white dwarfs as progenitors of R Coronae Borealis stars and Type I supernovae, The Astrophysical Journal 277 (1984) 355.

[6] K. Nomoto, Accreting white dwarf models for type 1 supernovae. II - Off-center detonation supernovae, The Astrophysical Journal 257 (1982) 780.

[7] W. Hillebrandt, M. Kromer, F. K. Röpke and A. J. Ruiter, Towards an understanding of Type Ia supernovae from a synthesis of theory and observations, Frontiers of Physics 8 (2013) 116 $[1502.6420]$.

[8] K. Maeda and Y. Terada, Progenitors of type Ia supernovae, Enternational Journal of Modern Physics D25(2016) 1630024 [1609.03639].

[9] W. Li, J. S. Bloom, P. Podsiadlowski, A. A. Miller, S. B. Cenko, S. W. Jha et al., Exclusion of a luminous red giant as a companion star to the progenitor of supernova sn 2011 fe, Nature $\mathbf{4 8 0}$ (2011) B48.

[10] P. L. Kelly, O. D. Fox, A. V. Filippenko, S. B. Cenko, L. Prato, G. Schaefer et al., Constraints on the progenitor system of the type ia supernova $2014 \mathrm{j}$ from pre-explosion hubble space telescope imaging, The Astrophysical Journal 790 (2014)3.

[11] B. E. Schaefer and A. Pagnotta, An absence of ex-companion stars in the type Ia supernova remnant SNR 0509-67.5, Nature 481 (2012) 164.

[12] H. Saio and K. Nomoto, Evolution of a merging pair of $C+O$ white $d$ warfs to form a single neutron star, Astronomy \& Astrophysics 150 (1985) L21.

[13] K. Nomoto and Y. Kondo, Conditions for Accretion-induced Collapse of White Dwarfs, the Astrophysical Journal Letters 367 (1991) L19.

[14] R. Pakmor, M. Kromer, F. K. Röpke, S. A. Sim, A. J. Ruiter and W. Hillebrandt, Sub-luminous type Ia

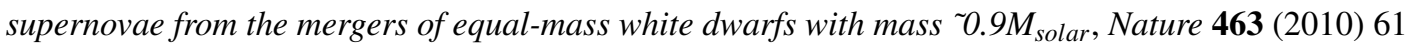
[0911.0926].

[15] S. Rosswog, D. Kasen, J. Guillochon and E. Ramirez-Ruiz, Collisions of white dwarfs as a new progenitor channel for type ia supernovae, The Astrophysical Journal Letters 705 (2009) L128 [0907.3196].

[16] C. Raskin, E. Scannapieco, G. Rockefeller, C. Fryer, S. Diehl and F. X. Timmes, ${ }^{56} \mathrm{Ni}$ Production in Double-degenerate White Dwarf Collisions, The Astrophysical Journal 724(2010)111 [1009.2507].

[17] P. Lorén-Aguilar, J. Isern and E. García-Berro, Smoothed particle hydrodynamics simulations of white $d$ warf collisions and close encounters, Monthly Notices of the Royal Astronomical Society 406 (2010) [2749 [1004.4783]. 
[18] J. Guillochon, M. Dan, E. Ramirez-Ruiz and S. Rosswog, Surface Detonations in Double Degenerate Binary Systems Triggered by Accretion Stream Instabilities, The Astrophysical Journal Letters 709 [2010) L64 [0911.0416].

[19] R. Pakmor, M. Kromer, S. Taubenberger and V. Springel, Helium-ignited Violent Mergers as a Unified Model for Normal and Rapidly Declining Type Ia Supernovae, The Astrophysical Journat Letters 770 (2013) L8] [15302.2913].

[20] K. J. Shen, D. Boubert, B. T. G"ansicke, S. W. Jha, J. E. Andrews, L. Chomiuk et al., Three hypervelocity white dwarfs in gaia dr2: Evidence for dynamically driven double-degenerate double-detonation type ia supernovae, The Astrophysical Journal 865 (2018) 15 [ए804. 11163$]$ ].

[21] R. Kashyap, R. Fisher, E. García-Berro, G. Aznar-Siguán, S. Ji and P. Lorén-Aguilar, Spiral instability can drive thermonuclear explosions in binary white dwarf mergers, The Astrophysical Dournal Letters 800 (2015) L7] [1501.05645].

[22] D. Fenn, T. Plewa and A. Gawryszczak, No double detonations but core carbon ignitions in high-resolution, grid-based simulations of binary white dwarf mergers, Monthly Notices of the Royat Astronomical Society 462 (2016) 2486 [1611.05730].

[23] A. Tanikawa, N. Nakasato, Y. Sato, K. Nomoto, K. Maeda and I. Hachisu, Hydrodynamical Evolution of Merging Carbon-Oxygen White Dwarfs: Their Pre-supernova Structure and Observational Counterparts, The Astrophysical Journal 807 (2015) 40 [15504.06035].

[24] Y. Sato, N. Nakasato, A. Tanikawa, K. Nomoto, K. Maeda and I. Hachisu, A Systematic Study of Carbon-Oxygen White Dwarf Mergers: Mass Combinations for Type Ia Supernovae, The Astrophysical Journal 807 (2015) 105 [4505.01646].

[25] Y. Sato, N. Nakasato, A. Tanikawa, K. Nomoto, K. Maeda and I. Hachisu, The Critical Mass Ratio of Double White Dwarf Binaries for Violent Merger-induced Type Ia Supernova Explosions, the Astrophysical Journal 821 (2016)67 [1603.01088].

[26] R. Pakmor, P. Edelmann, F. K. Röpke and W. Hillebrandt, Stellar GADGET: a smoothed particle hydrodynamics code for stellar astrophysics and its application to Type Ia supernovae from white dwarf mergers, Monthly Notices of the Royal Astronomical Society 424 (2012) 2222 [11205.5806].

[27] P. E. Nugent, M. Sullivan, S. B. Cenko, R. C. Thomas, D. Kasen, D. A. Howell et al., Supernova SN 2011 fe from an exploding carbon-oxygen white dwarf star, Nature 480 (2011) 344 [ [1110.6201].

[28] J. S. Bloom, D. Kasen, K. J. Shen, P. E. Nugent, N. R. Butler, M. L. Graham et al., A Compact Degenerate Primary-star Progenitor of SN 2011 fe, The Astrophysical Journal Letters 744(2012) L17] [LIII.0966].

[29] E. Livne, Successive detonations in accreting white dwarfs as an alternative mechanism for type I supernovae, The Astrophysical Journal Letters 354 (1990) L53.

[30] A. Tanikawa, K. Nomoto and N. Nakasato, Three-dimensional Simulation of Double Detonations in the Double-degenerate Model for Type Ia Supernovae and Interaction of Ejecta with a Surviving White Dwarf Companion, The Astrophysical Journal 868(2018)90] [11808.01545].

[31] A. Tanikawa, K. Nomoto, N. Nakasato and K. Maeda, Double-detonation Models for Type Ia Supernovae: Trigger of Detonation in Companion White Dwarfs and Signatures of Companions' Stripped-off Materials, The Astrophysical Journal 885 (2019) 103 [1009.09770]. 